\title{
Ambulatório de diabetes mellitus: ações de enfermagem na atenção secundária em saúde
}

\author{
Raíla S. Santos, ${ }^{1,2 *}$ Amanda V. Torres ${ }^{1,2}$
}

\section{Resumo}

Este relato de experiência tem como objetivo descrever a implementação do serviço e as ações da equipe de enfermagem em um ambulatório de diabetes. O ambulatório faz parte do conjunto de clínicas de uma policlínica de assistência secundária da Universidade do Estado do Rio de Janeiro (UERJ) e oferece atendimento integral com equipe multiprofissional (médico, enfermeiro, psicólogo, nutricionista, fisioterapeuta, pedagogo e educador físico) para usuários com diagnóstico de diabetes mellitus tipo 1, tipo 2 e diabetes gestacional. A equipe de enfermagem do ambulatório se integra a uma equipe multiprofissional e orienta as ações no cuidado ao paciente diabético a partir das diretrizes para o cuidado das pessoas com doenças crônicas. A consulta de enfermagem, as ações educativas (sala de espera e grupos) e a realização de retinografia para rastreamento da retinopatia diabética (RD) estão entre as principais atividades desenvolvidas pela equipe de enfermagem do ambulatório para o acompanhamento do paciente diabético. Em relação à retinografia, temos experiência exitosa no ambulatório, com a finalidade de prevenir e melhor tratar a retinopatia diabética, a fim de proporcionar maior qualidade de vida ao paciente diabético e menor custo social, evitando longas filas e aumentando a realização do exame para pacientes que nunca tinham realizado. A equipe de enfermagem, portanto, busca disseminar orientações que estimulam o usuário a buscar o autocuidado diário, promovendo uma melhor qualidade de vida e maior autonomia no controle e tratamento do diabetes.

Descritores: Diabetes mellitus; Retinopatia diabética; Educação em saúde; Atenção secundária à saúde; Cuidados de enfermagem.

\section{Abstract \\ Diabetes mellitus clinic: nursing actions on secon- dary health care}

This experience report aimed to describe the implementation of the service and the nursing staff and their actions in a diabetes clinic. The clinic is part of the set of clinics secondary care polyclinic of the Universidade do Estado do Rio de Janeiro (UERJ) and provides comprehensive care with multidisciplinary staff (doctor, nurse, psychologist, dietitian, physical therapist, teacher and physical education) for users diagnosed with diabetes mellitus type 1, type 2 and gestational diabetes. The clinic's nursing staff is integrated to a multidisciplinary team and guides the actions in the care
1. Programa de Pós-Graduação em Ciências Médicas. Universidade do Estado do Rio de Janeiro.

2. Serviço de Diabetes. Policlínica Piquet Carneiro. Universidade do Estado do Rio de Janeiro. Rio de Janeiro, RJ, Brasil.

\section{*Endereço para correspondência:}

Serviço de Diabetes, Policlínica Piquet Carneiro, UERJ

Avenida Marechal Rondon, 381

Rio de Janeiro, RJ, Brasil. CEP: 20950-003.

E-mail: railappc@gmail.com

Revista HUPE, Rio de Janeiro, 2015;14(4):81-84

doi: 10.12957/rhupe.2015.20063

Recebido em 28/11/2014. Aprovado em 09/01/2015.

of diabetic patients from the guidelines for the care of people with chronic diseases. The nursing consultation, educational activities (waiting room and groups) and performing retinography for screening of diabetic retinopathy (DR) are among the main activities developed by ambulatory nursing staff for the diabetic patient monitoring. About retinography, we have successful experience in the clinic, in order to prevent and treat diabetic retinopathy in order to provide better quality of life for diabetics patients, and lower social cost avoiding long lines and increasing the exam for patients who had never done. The nursing staff, therefore, seeks to disseminate guidelines that encourage the user to get the daily self-care, promoting a better quality of life and greater autonomy in the control and treatment of diabetes.

Keywords: Diabetes mellitus; Diabetic retinopathy; Health education; Secondary care; Nursing care.

\section{Resumen}

\section{Centro de salud de diabetes mellitus: acciones de} enfermería en la atención secundaria en salud

Este relato de experiencia tiene como objetivo describir la implementación del servicio y las acciones del equipo de enfermería en un centro de salud de diabetes. El centro de salud es parte del conjunto de clínicas de una policlínica de atención secundaria de la Universidad del Estado de Río de Janeiro (UERJ) y proporciona atención integral con equipo multidisciplinario (médico, enfermero, psicólogo, nutricionista, fisioterapeuta, pedagogo y instructor físico) para los usuarios diagnosticados con diabetes mellitus tipo 1, tipo 2 
y diabetes gestacional. El personal de enfermería del centro de salud se integra a un equipo multidisciplinario y guía las acciones en el cuidado de los pacientes diabéticos, a partir de las directrices para el cuidado de personas con enfermedades crónicas. La consulta de enfermería, las actividades educativas (sala de espera y grupos) y la realización de la retinografía para el seguimiento de la retinopatía diabética (RD) se encuentran entre las principales actividades desarrolladas por el equipo de enfermería del centro de salud para el seguimiento del paciente diabético. Con relación a la retinografía, tenemos experiencia exitosa en el centro de salud, con el fin de prevenir

\section{Introdução}

No Brasil, as doenças crônicas não transmissíveis (DCNT) são responsáveis por $72 \%$ dos óbitos. Dentre as principais DCNT destacam-se as doenças do aparelho circulatório, câncer, diabetes e doenças respiratóriocrônicas. ${ }^{1,2}$

O diabetes mellitus (DM) é uma doença metabólica grave de etiologia múltipla, caracterizada por hiperglicemia crônica decorrente da falta de insulina e/ou a incapacidade de insulina para exercer adequadamente seus efeitos. ${ }^{1,3}$

É uma doença de importância mundial que vem se tornando um problema de saúde pública, tomando proporções crescentes no que se refere ao aparecimento de novos casos. É uma das principais doenças crônicas que afetam o homem acometendo populações de países em todos os estágios de desenvolvimento econômicosocial. ${ }^{2}$ Vários órgãos podem ser acometidos pelo DM, sendo comum a presença de nefropatia, neuropatia e retinopatia., ${ }^{1,3}$

ODM compreende quatro categorias: diabetes tipo 1; diabetes tipo 2; diabetes gestacional e outros. Os tipos mais frequentes são o diabetes tipo 1, que representa cerca de $10 \%$ do total de casos e o diabetes tipo 2, conhecido como diabetes do adulto, que compreende cerca de $90 \%$ do total de casos. ${ }^{1}$

São vários os custos do Sistema Único de Saúde (SUS) com o paciente diabético, como: consultas de cuidados primários, consultas especializadas, internações hospitalares, atendimento de urgências e emergências, medicamentos orais, insumos para insulinoterapia (tiras-teste, seringas/agulhas, lancetas), custo com próteses relacionadas às amputações e exames laboratoriais. Em relação a custos mais significativos, destaca-se a hemodiálise pela insuficiência renal crônica, custos com tratamentos de fotocoagulação para os doentes com retinopatia diabética e outros. ${ }^{3}$ y tratar mejor la retinopatía diabética, para proporcionar una mayor calidad de vida al paciente diabético y un menor costo social, evitando las largas filas y aumentando la realización del examen para los pacientes que nunca lo hubiesen hecho. El personal de enfermería, por lo tanto, busca difundir las orientaciones que fomenten al usuario a obtener el autocuidado diario, promoviendo una mejor calidad de vida y una mayor autonomía en el control y tratamiento de la diabetes.

Palabras clave: Diabetes mellitus; Retinopatía diabética; Educación en salud; Atención secundaria de salud; Cuidados de enfermería.

O ambulatório de diabetes faz parte do conjunto de clínicas de uma policlínica de assistência secundária da Universidade do Estado do Rio de Janeiro (UERJ) e oferece atendimento integral com equipe multiprofissional (médico, enfermeiro, psicólogo, nutricionista, fisioterapeuta, pedagogo e educador físico) para usuários com diagnóstico de diabetes mellitus tipo 1, tipo 2 e diabetes gestacional.

A equipe de enfermagem do ambulatório se integra a uma equipe multiprofissional e orienta as ações no cuidado ao paciente diabético a partir das diretrizes para o cuidado das pessoas com doenças crônicas através das redes de atenção à saúde e pelas linhas de cuidado prioritárias. ${ }^{2}$

As atividades desenvolvidas pela equipe de enfermagem para o acompanhamento do paciente diabético englobam: consulta de enfermagem, ações educativas (sala de espera e grupos), supervisão de estagiários e internos dos cursos enfermagem, pesquisa em enfermagem baseada em indicadores e a realização de retinografia para rastreamento da retinopatia diabética (RD).

Este relato de experiência tem como objetivo descrever a implementação do serviço e as ações da equipe de enfermagem em um ambulatório de diabetes da atenção secundária do município do Rio de Janeiro.

\section{Ações de enfermagem na atenção ao paciente diabético}

O processo de trabalho da equipe de enfermagem do ambulatório de diabetes baseia-se na promoção da saúde, prevenção e tratamento do DM tipo 1, tipo 2 e diabetes gestacional. Dessa forma, uma das principais atividades da equipe de enfermagem é a educação em saúde com foco nas mudanças do estilo de vida, prevenção de complicações agudas e crônicas da doença e orientações sobre o tratamento. 
O atendimento ao paciente diabético pela equipe de enfermagem se desenvolve de acordo com as seguintes temáticas: "Acolhimento no primeiro atendimento ao paciente com diagnóstico de diabetes"; "Intervenção para mudança de hábitos de vida em diabetes: alimentação saudável, atividade física e tabagismo"; "Autocuidado apoiado em diabetes: complicações agudas (hiperglicemia e hipoglicemia)"; "Autocuidado apoiado em diabetes: complicações crônicas"; "Autocuidado apoiado em diabetes: cuidado com os pés"; "Autocuidado apoiado em diabetes: uso correto da medicação oral e insulina e medicação da glicemia capilar"; "Avaliação e acompanhamento dos pacientes em uso de insulinoterapia".

A sala de espera realizada pela equipe de enfermagem tem como foco a prevenção de complicações crônicas e agudas, o aumento da adesão ao tratamento e a promoção da qualidade de vida. Um projeto educativo precisa visar o autoconhecimento e facilitar a troca de experiências e habilidades entre paciente e profissional através do processo de ensino-aprendizagem. $\mathrm{O}$ paciente deve ser inserido como sujeito integrante no seu tratamento, sendo corresponsável pelo cuidado junto com os profissionais de saúde. Além de todos os benefícios identificados para o paciente, a sala de espera proporciona maior integração da equipe e estabelece vínculos efetivos entre profissionais e pacientes.

A atuação da equipe de enfermagem na sala de espera é fator determinante no processo de educação em saúde dos pacientes. No período de um ano foram realizadas, aproximadamente, 58 reuniões em salas de espera, em média cada uma com a participação de 15 pacientes. Alguns temas trabalhados nas salas de espera de enfermagem: "Insulinoterapia e monitorização da glicemia capilar"; "Aprendendo a reconhecer hipoglicemias e hiperglicemias"; "Cuidados com o pé diabético".

Além das atividades assistenciais e educativas, o setor está comprometido em estruturar o campo de estágio (ensino) para treinamento, capacitação e desenvolvimento de recursos humanos na área da saúde. A equipe de enfermagem, portanto, busca disseminar orientações que estimulam o usuário a buscar o autocuidado diário, através de atitudes simples, como verificar e cuidar dos pés, reeducação alimentar, aplicação correta da insulina e uso correto da medicação oral. O paciente passa a ter uma constante autovigilância sobre o seu corpo e sua saúde, promovendo uma melhor qualidade de vida e maior autonomia no controle e tratamento da doença. 4,5

\section{A enfermagem na tecnologia diagnóstica: retinografia}

A retinopatia diabética (RD) é a principal causa de cegueira entre diabéticos. Esta complicação ocorre devido à grande concentração de glicose na circulação sanguínea, o que lesa os vasos sanguíneos que nutrem a retina. A diminuição da disponibilidade de vasos fisiologicamente normais que possam nutrir e oxigenar a retina provoca hipóxia tecidual, resultando em uma diminuição da acuidade visual ao longo dos estágios da doença. ${ }^{6}, 7$

É estimado atualmente um número aproximado de dois milhões de brasileiros com RD e que $50 \%$ dos pacientes portadores de DM sejam afetados pela RD, sendo responsável por 7,5\% das causas de incapacidade de adultos para a prática do trabalho e por $4,58 \%$ das deficiências visuais. ${ }^{6,8}$

Idealmente, o rastreamento para $\mathrm{RD}$ deveria ser realizado por oftalmologista da rede pública, mas este sistema tem-se mostrado insuficiente, na medida em que apenas uma pequena porção dos pacientes tem acesso ao especialista. ${ }^{6}$ A quantidade de centros com equipamentos e profissionais aptos para o diagnóstico ainda é considerada insuficiente. No Brasil, estima-se que existam 48.707 ambulatórios oftalmológicos, mas apenas 7\% são credenciados ao SUS. ${ }^{8}$

Para otimizar a realização de retinografia, as imagens da retina são realizadas através de sistemas digitais, que podem ser visualizadas e armazenadas em computador. ${ }^{6,7} \mathrm{~A}$ captura da imagem com recursos diagnósticos pode ser realizada por profissional de nível médio treinado. No ambulatório o exame é realizado pela enfermeira e técnica de enfermagem, que foram treinadas pela oftalmologista. Posteriormente, é analisado por oftalmologista para emissão do laudo.

Em relação à realização de retinografia pela equipe de enfermagem do ambulatório, foi identificado aumento expressivo da captação de pacientes com DM tipo 2 (134 exames em três meses). Destes, 86 eram do sexo feminino e 48 do sexo masculino, apenas 18 fotografias não foram satisfatórias com relação à qualidade diagnosticada pelo profissional médico oftalmologista.

É preciso que haja ações coordenadas com o objetivo de evitar a cegueira ou diminuir as complicações da doença, principalmente no Brasil, onde cerca de 70\% da população depende do SUS. O objetivo da inserção da enfermagem na utilização das tecnologias diagnósticas como a retinografia é otimizar o processo de realização do exame, para reduzir as filas de espera, diminuindo as 
estatísticas de perda da visão em relação ao tempo de doença e a progressão da retinopatia diabética.

\section{Conclusão}

O tratamento do DM é complexo. O primeiro passo terapêutico envolve mudanças no estilo de vida (dieta saudável e atividade física). Os efeitos benéficos e a elevada eficácia dessa estratégia sustentam melhor controle glicêmico, perda de peso e aumento da sensibilidade à insulina, que reduz a necessidade de tratamento medicamentoso.

As intervenções de enfermagem aos pacientes com diabetes são abrangentes e justificadas pela complexidade do tratamento desta doença. ${ }^{4}$ As orientações individuais e em grupos (sala de espera) são iniciadas de acordo com as necessidades dos pacientes e proporcionam um espaço de envolvimento com a saúde e o bem-estar, resultando na construção de vínculos que visam promover melhor qualidade de vida, prevenção de complicações crônicas e agudas e adesão ao tratamento. ${ }^{4,6}$

Em relação à retinografia, temos experiência exitosa no ambulatório, através da realização do exame pela equipe de enfermagem capacitada por um oftalmologista, evitando longas filas e aumentando a realização do exame para pacientes que nunca tinham realizado. Todos esses esforços com novas modalidades de tratamento têm o objetivo de prevenir e melhor tratar a retinopatia diabética, a fim de proporcionar maior qualidade de vida ao paciente diabético e menor custo social.

Relatos de experiência em serviço de saúde propiciam a divulgação do processo de trabalho desenvolvido e podem ser usados como exemplos para imple- mentação de novos serviços no âmbito da atenção secundária. A atuação da equipe de enfermagem no ambulatório de diabetes é positiva e fundamental para o controle do diabetes. Cabe à enfermagem estimular o autocuidado, promover a autonomia e reconhecer o paciente diabético como sujeito corresponsável no controle e tratamento de sua doença.

\section{Referências}

1. Standards of medical care in diabetes--2014. Diabetes Care. 2014 Jan;37 Suppl 1:S14-80.

2. Brasil. Ministério da Saúde. Secretaria de Atenção à Saúde. Departamento de Atenção Básica. Diabetes Mellitus. Brasília: Ministério da Saúde; 2006. 64p. (Cadernos de Atenção Básica $n^{\circ}$ 16. Série A. Normas e Manuais Técnicos.)

3. Brasil. Ministério da Saúde. Secretaria de Atenção à Saúde. Departamento de Atenção Básica. Diretrizes para o cuidado das pessoas com doenças crônicas nas redes de atenção à saúde e nas linhas de cuidado prioritárias. Brasília: Ministério da Saúde; 2014. (Cadernos de Atenção Básica n 35. Série A. Normas e Manuais Técnicos.)

4. Freitas RWJF, Araújo MFM, Marinho NBP, et al. Fatores relacionados ao diagnóstico de enfermagem autocontrole ineficaz da saúde entre diabéticos. Acta Paulista de Enfermagem. 2011;24(3):365-72.

5. Chaves Mde O, Teixeira MR, da Silva SE. Perceptions of people with diabetes about the disease: nursing contributions. Rev Bras Enferm. 2013 Mar-Apr;66(2):215-21.

6. Gross JL, Nehme M. Detection and treatment of chronic complications of diabetes mellitus: Consensus of the Brazilian Diabetes Society and the Brazilian Ophthalmology Council. Rev Assoc Med Bras. 1999 Jul-Sep;45(3):279-84.

7. Klein R, Klein BE, Moss SE, et al. The Wisconsin epidemiologic study of diabetic retinopathy. II. Prevalence and risk of diabetic retinopathy when age at diagnosis is less than 30 years. Arch Ophthalmol. 1984 Apr;102(4):520-6.

8. Maia O O Jr, Marback RF, Bonanomi MT, et al. Delay in ophthalmologic examination of diabetic retinopathy patients. Rev Assoc Med Bras. 2007 Jan-Feb;53(1):39-43. 